\title{
Pesquisando sobre Transparência: Resultados Preliminares de um Mapeamento Sistemático
}

\author{
Elizabeth Suescún Monsalve ${ }^{1}$, Julio Cesar Sampaio do Prado Leite ${ }^{2}$ \\ ${ }^{1}$ Departamento de Sistemas e Informática - Universidad EAFIT - Carrera 49 \# 7 sur - \\ 50, Medellín, Antioquia, Colombia \\ ${ }^{2}$ Departamento de Informática, Pontifícia Universidade Católica do Rio de Janeiro, \\ Rua Marquês de São Vicente 225, Ed. Padre Leonel Franca 13o. andar, \\ Rio de Janeiro, Brasil \\ Esuesculdeafit.edu.co, http://www-di.inf.puc-rio.br/ julio
}

\begin{abstract}
This paper explores the use of systematic review in the area of transparency, with a particular emphasis on software related transparency. It lays out the overall steps of a systematic review according to the literature, and presents initial results, of an exploratory nature. One of the results so far, is the discovery of the lack secondary studies, which points out to novelty of this presentation.
\end{abstract}

Resumo. Na Engenharia de Software, nos últimos anos, tem-se observado um crescente interesse em conduzir estudos empíricos que ajudem a entender, sob certas condições, um domínio ou tema de pesquisa. $O$ objetivo deste artigo é apresentar o protocolo seguido no intuito de desenvolver um Estudo de Mapeamento Sistemático para o tema de transparência para trabalhos publicados em Inglês. Além disso, já se apresentam os primeiros resultados da abordagem, que objetiva identificar as pesquisas e os trabalhos que permitem conhecer o que foi publicado sobre esse tema. Como limitações, encontramos que na primeira busca automática identificou-se a falta de estudos secundários no contexto da transparência. Para concluir, acredita-se que no contexto da transparência estudos de mapeamento podem economizar tempo e esforço dos pesquisadores, fornecer orientações, descrever lacunas e ajudar nos novos esforços de pesquisa.

\section{Introdução}

Um mapeamento sistêmico de literatura ou Estudos de Mapeamento Sistemático (EMS) como é explicado por Kitchenham e Charters (2007) e Petersen e outros (2008) é um estudo que permite dar uma visão geral de uma área de pesquisa através da classificação e contribuições a partir de estudos primários. Nesse contexto, EMS, um estudo secundário, é o resultado de um mapeamento sistemático, que envolve buscas na literatura para identificar que tópicos têm sido estudados numa área de pesquisa e quais veículos publicam esses estudos primários.

Na engenharia de software os EMS são usados para estruturar áreas de pesquisa. Neste trabalho foram estudadas as abordagens propostas por Kitchenham e Charters (2007) e Levy e Ellis (2006) os quais propõem protocolos para guiar o mapeamento. Para nosso 
tópico de interesse não foram encontrados estudos secundários que poderiam complementar o trabalho a expor aqui.

Organizamos o artigo da seguinte maneira: na Seção 2 são apresentados os trabalhos relacionados com estudos empíricos e a motivação. Na Seção 3 é descrito o processo de mapeamento sistemático proposto nesse estudo. Na Seção 4 são apresentados os objetivos e questões de pesquisa. A Seção 5 descreve a estratégia de busca inicial. Finalmente, são apresentados os resultados já alcançados, bem como futuras pesquisas.

\section{Trabalhos Relacionados}

Ao longo dos últimos anos observa-se que Engenharia de Software (ES) como outras áreas do conhecimento (ex: Ciências Sociais e a Medicina), tem mostrado interesse em conduzir estudos empíricos. Segundo De Almeida Filho (2011) esse tipo estudo tem evidenciado sua relevância para o avanço da ciência e servem como instrumento para o desenvolvimento de uma área que é fortemente dependente das atividades humanas. A motivação para desenvolver estudos empíricos é a identificação de lacunas, as quais são importantes para propor estudos, construir o corpo de conhecimento e apoiar na tomada de decisões por parte de profissionais e pesquisadores.

Kitchenham et al (2005) escreveram que o conceito prática baseada em evidência que já era usada na medicina e adaptada em outras disciplinas como a economia, psicologia, e ciências sociais, deveria ser adaptado na ES, para criar a Engenharia de Software Baseada em Evidencias (ESBE); para isso deveriam ser usadas as revisões de literatura como uma metodologia para obter resultados empíricos. Os mesmos autores explicam que ESBE deveria aplicar quatro estágios.

Primeiro, construir uma pergunta de pesquisa, segundo, trazer os resultados conforme a pergunta de pesquisa, terceiro, avaliar de forma critica as evidencias, e finalmente, usar a evidencia para direcionar a pesquisa. Como foi mencionado EBSE é subjacente a procedimentos de busca de literatura chamados Revisão Sistemática de Literatura (RSL) e Estudos de Mapeamento Sistemático EMS, ambos procedimentos que usam protocolos. No entanto, o primeiro possui uma pergunta especifica que direciona $o$ estudo, o segundo, revisa de forma ampla um tópico e classifica os estudos primários para esse domínio especifico.

\section{O Processo do Mapeamento Sistemático}

Para o presente trabalho, limitado a literatura na língua inglesa, foram usadas as diretrizes propostas pelo protocolo para mapeamento sistemático proposto por Petersen e outros (2008), os autores comparam os métodos usados nos mapeamentos sistemáticos (EMS) e nas revisões sistemáticas de literatura (RSL). São propostos cinco estágios para direcionar um EMS, assim: 1. Definir a pergunta de pesquisa; 2. Direcionar os estudos primários, Keele (2007) define como estudos primários (no contexto da engenharia de software baseada em evidência) estudos empíricos que trabalham sobre uma pergunta de pesquisa específica e podem ser do tipo: experimento controlado, estudo de caso, revisão de literatura, etnografia e pesquisa ação; 3. Fazer triagem dos artigos baseados nos critérios de inclusão e de exclusão; 4. Fazer a classificação dos artigos, e finalmente; 5. Extrair e mapear a coleção de dados. Ressaltando, que cada estágio produz um resultado usado como entrada para o seguinte estagio. 


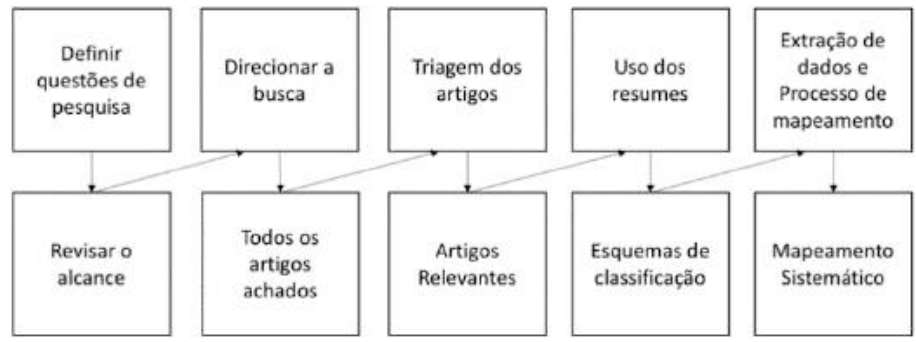

Figura 1. Processo de Mapeamento Sistemático

Fonte: Petersen e outros (2008)

\section{Questões de Pesquisa}

As perguntas de pesquisa foram elaboradas seguindo a abordagem proposta por Basili e outros (2012), conhecida com Goal-Question-Metrics (GQM). Essa abordagem do tipo de cima para baixo, tem como objetivo desenvolver métricas significativas através de perguntas. Conforme é descrito por Yang e outros (2014) esta abordagem compreende: 1. O Objetivo está no nível conceptual, define o objeto e a variedade de ações, variedade de modelos, pontos de vista, o relativo e o ambiente particular; 2. A Questão está no nível operacional, as perguntas são usadas para definir o modelo do objeto de estudo e assim focar esse objeto nas características da avaliação e objetivos específicos a serem atingidos; finalmente, 3. A Métrica está no nível quantitativo, nele temos um conjunto de métricas baseadas nos modelos as quais podemos associar a cada questão visando a resposta medível de cada um.

O objetivo de alto nível neste estudo de mapeamento sistemático para transparência é identificar as pesquisas relacionadas, para assim poder responder a pergunta $O$ que nós sabemos sobre o Tema Transparência? Para atingir esse objetivo, nós listamos quatro sub-objetivos operacionalizáveis.

G1: Fornecer as informações básicas, delimitar o escopo desde a perspectiva demográfica e permitir identificar as fontes pertinentes sobre informação de pesquisa na área de Transparência.

G2: Ajudar pesquisadores e interessados sobre as propostas a nível de qualidade, validação e processos usados nas abordagens dos autores selecionados.

G3: Identificar que propostas são mais maduras quanto aos resultados alcançados.

G4: Identificar as principais tendências de pesquisa, trabalhos futuros, trabalhos em andamento relacionados a transparência.

As metas acima descritas em conjunto com as justificativas servem de guia nas questões de pesquisa assim: 14 perguntas medíveis e/o respondíveis (RQ) na tabela $1 \mathrm{em}$ conjunto com as motivações Tabela 2. Em conjunto é possível mapear cada meta com a sub-meta e a motivação. 
Tabela 1. As perguntas de pesquisa

\begin{tabular}{|l|l|l|}
\hline Questões de Pesquisa & Tipo & $\begin{array}{l}\text { Objetivo } \\
\text { Relacionado }\end{array}$ \\
\hline $\begin{array}{l}\text { RQ1: Qual é a distribuição de tempo dos estudos primários? } \\
\text { RQ2: Qual é distribuiçăo local dos estudos primários? } \\
\text { RQ3: Qual é a distribuição geográfica dos estudos primários? } \\
\text { RQ4: Quais são os estudos primários mais citados? }\end{array}$ & Publicação & G1 \\
\hline $\begin{array}{l}\text { RQ5: Existe um consenso sobre a definição de transparência? } \\
\text { RQ6: Que processos de Engenharia de Requisitos são usados? } \\
\text { RQ7: Que domínios são usados para ilustrar a aplicação do conceito? } \\
\text { RQ8: Que atributos de qualidade são propostos? }\end{array}$ & Conteúdo & G2 \\
\hline $\begin{array}{l}\text { RQ9: Quais métodos têm sido aplicados e a rigorosidade a avaliação? } \\
\text { RQ10: Que resultados já foram alcançados? }\end{array}$ & Qualidade & G3 \\
\hline $\begin{array}{l}\text { RQ11: Que tópicos podemos obter dos estudos selecionados? } \\
\text { RQ12: Que métodos e/ou processos são usados nos tópicos encontrados? } \\
\text { RQ13: Qual é a tendencia para o tópico de Transparencia na ultima década? }\end{array}$ & Tópico & G4 \\
\hline
\end{tabular}

Tabela 2. Motivação perguntas de pesquisa

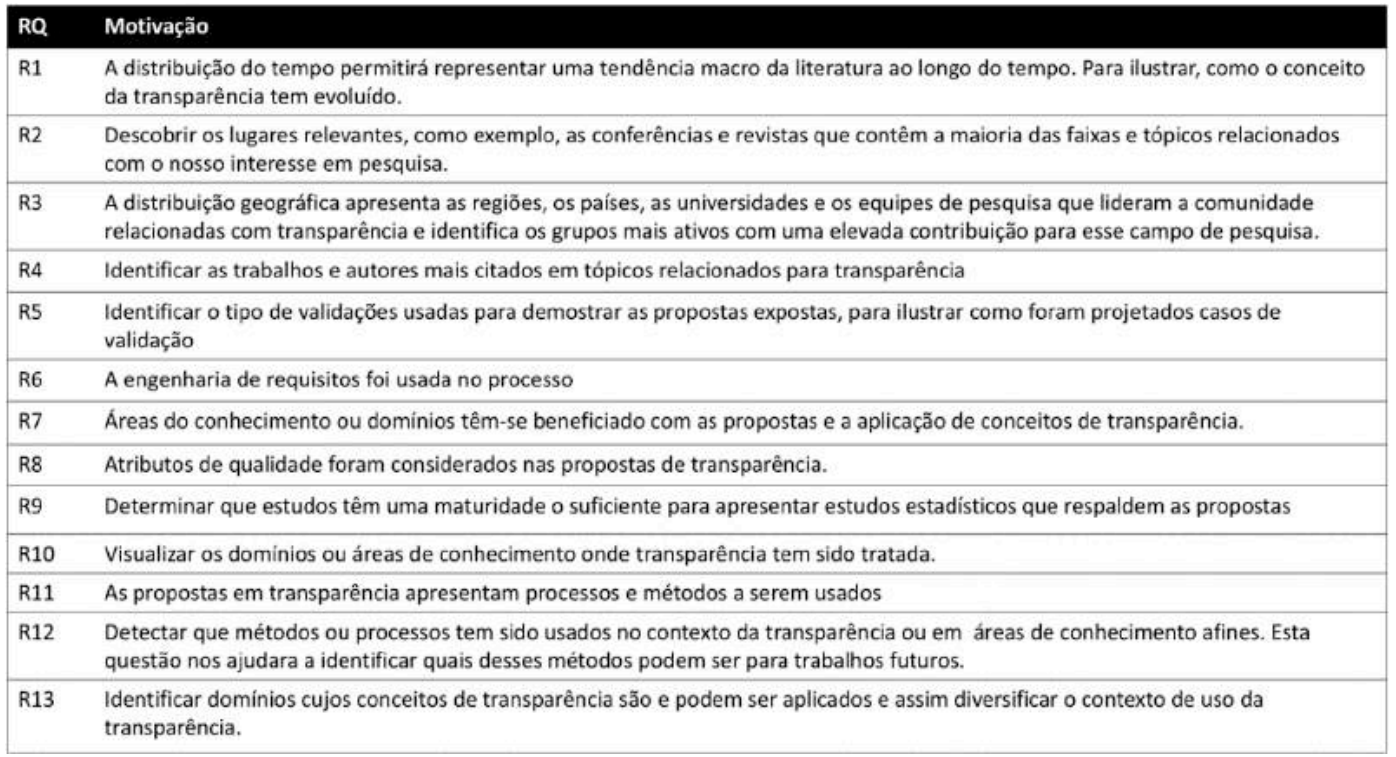

As Métricas da proposta GQM, serão desenvolvidas em trabalhos futuros.

\section{Estratégia de Busca com critérios de seleção}

\subsection{O Quasi Gold Standard}

O objetivo do processo de busca é identificar estudos relevantes que tratem as questões de pesquisa acima descritas. A estratégia de busca compreende identificar as fontes de busca e definir os Strings de busca. Para o alcance deste trabalho preliminar será explicada a estratégia e se detalhará a busca automática baseada nos itens aqui expostos.

A estratégia de busca é baseada na proposta de a qual descreve aquilo que os autores chamam de Quasi Gold Standard QGS. Essa proposta tem como objetivo projetar e executar estratégias para extração de dados com foco em RSL (ver a Figura 2), no entanto, os conceitos propostos podem ser aplicados para EMS. Conforme os autores o Quasi Gold é um conjunto conhecido de estudos primários tão fiel quanto possível para aquelas perguntas de pesquisa propostas para direcionar a RLS. Neste contexto, esta abordagem do QGS permite a recuperação e identificação de estudos relevantes em engenharia de software e nosso caso especifico: transparência. Segundo esses autores, Zhang et al (2011), um QGS é um conjunto de estudos conhecidos encontrados com 
uma pesquisa manual dos locais relacionados (onde foram publicados) para um determinado período de tempo (período). Um QGS pode ser considerado um "padrãoouro" nas condições em que o período e o local se aplicam.

Continuando com a explicação dos autores, o QGS é útil no sentido de tornar mais objetivo o processo desde a perspectiva do planejamento, teste manuais e Strings para extração de dados. A ideia principal é evitar a falta de estudos relevantes na estratégia de busca. Portanto, os resultados das pesquisas manuais e automatizadas são comparados com o QGS para calcular a Sensibilidade (Número de estudos relevantes recuperados / Número total de estudos relevantes) *100) e Precisão (Número de estudos relevantes recuperados / Número de estudos recuperados) *100) dos resultados da pesquisa. Alta sensibilidade e alta precisão são desejáveis, mas uma alta sensibilidade é mais importante do que alta precisão. A sensibilidade acima de $80 \%$ é ideal para melhores resultados, mas os resultados entre $70 \%$ e $80 \%$ também são aceitáveis de acordo com Zhang et al. (2011).

Adicionalmente, o trabalho aqui apresentado também incorpora uma estratégia de snowballing para trás e para a frente (backward and forward snowballing) para expandir a cobertura de busca Wohlin (2014). Snowballing faz referência ao uso de listas de referencia dos artigos selecionados ou as citações desses artigos. Isso dizer que é possível achar ou refinar as buscas com os artigos que estão sendo citados ou identificar novos artigos que estão citando um artigo selecionado.

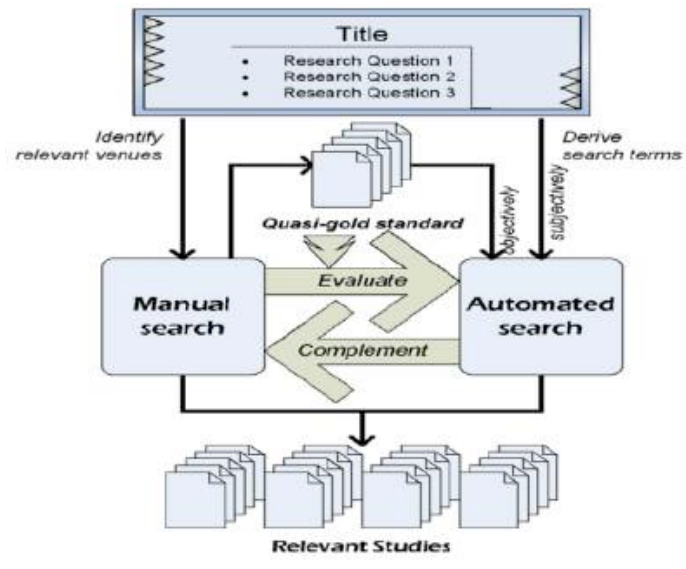

Figura 2. Estratégia de Busca Fonte: Wohlin (2014)

\subsection{Critérios de inclusão/exclusão}

Nosso objetivo geral é analisar e classificar os trabalhos que abordam o atributo de qualidade Transparência, no contexto de software. Portanto, vamos usar critérios de inclusão e exclusão na seleção de acordo. Primeiramente, será necessário verificar documentos usando os critérios de exclusão. Em seguida, se um artigo cumpre qualquer um dos critérios de exclusão, ele será excluído dos estudos relevantes. Por ultimo, os critérios de inclusão serão aplicados aos restantes artigos.

\section{Critérios de inclusão}

CI1. Artigos, capítulos de livros, dissertações, teses e capítulos de livros cujo domínio é ciência da computação, engenharia de software, transparência. 
CI 2. Artigos, capítulos de livros, dissertações, teses e capítulos de livros publicados entre janeiro de 1997 e março de 2017.

CI 3. Artigos, capítulos de livros, dissertações, teses e capítulos dos livros que falem de transparência como um atributo de qualidade.

CI 4. Artigos, capítulos de livros, dissertações, teses e capítulos de livros disponíveis em formato eletrônico.

\section{Os critérios de exclusão}

EC1. Relatórios técnicos, posters ou trabalhos que não foram publicados ainda.

EC2. Artigos que não tem o enfoque da transparência desde a perspectiva do cidadão.

EC3. Artigos que falam de transparência sem que esta tenha aplicação no contexto de software.

EC4. Abordagens sem detalhes sobre como aplicar o conceito da transparência.

\subsection{Seleção de documentos}

Para selecionar documentos relevantes vamos seguir o processo de triagem ou de seleção descrito na Figura 1 e a continuação se guiará pelo processo descrito na Figura 3. Esse processo tem quatro etapas: uma varredura por título, resumo, por conclusões e finalmente o texto completo. Cada varredura usa os critérios de inclusão e exclusão definidos acima. Será importante realizar todas as etapas e verificar os resultados obtidos.

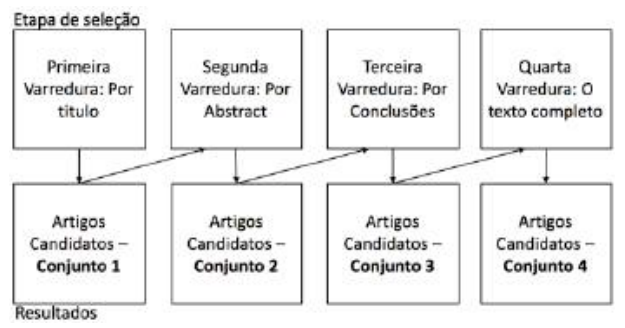

Figura 3. Processo de Seleção

- Fase 1: nessa primeira parte é possível analisar o titulo do artigo e as palavras-chave, com o objetivo de eliminar aqueles artigos irrelevantes. Todos aqueles artigos que sejam considerados que deve ser incluído devem permanecer no conjunto de documentos candidatos para a Fase 2.

- Fase 2: é necessário olhar e ler os resumos dos trabalhos candidatos da fase 1 e avaliar cada artigo com critérios de inclusão/exclusão. Todos aqueles artigos que sejam considerados que deve ser incluído e devem permanecer no conjunto de documentos candidatos para a Fase 3.

Fase 3: é necessário olhar e ler as conclusões dos trabalhos candidatos da fase 2 e avaliar cada artigo com critérios de inclusão/exclusão. Todos aqueles artigos que sejam considerados que deve ser incluído e devem permanecer no conjunto de documentos candidatos para a Fase 4.

- Fase 4: vamos ler os textos completos candidatos da Fase 3 e avaliar cada artigo com os critérios de inclusão/exclusão. Qualquer artigo que achemos que devem ser incluídos 
ou excluídos permanece no conjunto de estudos primários/secundários ou o conjunto de documentos excluídos.

\subsection{Artigos duplicados, estudos repetidos e estudos secundários}

Artigos duplicados são os mesmos artigos que retornam por diferentes estratégias de busca. Estudos duplicados são aqueles trabalhos sobre o mesmo tópico e que são publicados em diferentes veículos. Dessa maneira, excluem-se os artigos duplicados e os estudos repetidos. Por último, RSL ou EMS não estão incluídos em estudos relevantes. No entanto, eles são recuperados para validar o processo de pesquisa, através da comparação com os estudos primários incluídos. Importante ressaltar que esses estudos secundários são relevantes para a seção de trabalhos relacionados do relatório final do EMS em construção.

\subsection{Busca Automática}

\subsubsection{Motores de busca e bibliotecas digitais}

Para refinar a proposta aqui apresentada e passar para a segunda fase do trabalho, isto é a busca manual utilizamos uma busca automática inicial. Essa busca inicial contou com a ajuda do Descubridor da Universidade EAFIT (2017) e as bibliotecas digitais que ele agrupa. A Biblioteca da Universidade EAFIT oferece o serviço dessa busca automática inicial (Serviço de Buscas Universidade EAFIT). Para uso desse serviço descreve-se o propósito da busca, o String explicitado e participa-se de uma entrevista que visa definir o escopo e resumir o objetivo a ser alcançado. Com base nisso, o pessoal da Biblioteca realizou a primeira rodada com o String de busca proposto, que foi executado em: ACM Digital Library, British Standards Online, Directory of Open Access Journals, EBSCO Host, Emerald, IEEE Computer, Society Digital Library, ISI Web of Science, ProQuest, Safari entre outros. Adicionalmente, foram usados o Repositório da CEPAL e o Google Scholar. É importante ressaltar que o resultado dessa primeira rodada está supeditado ao entendimento que o pessoal da Biblioteca teve sobre o tópico de interesse.

\subsubsection{Strings de Busca}

O PICO (População, Intervenção, Comparação e Resultados) sugerido por Kitchenham e Charters (2077) tem como objetivo identificar palavras-chave e formular Strings de busca a partir de perguntas de pesquisa. A estratégia resumida na Figura 4 tem como objetivo identificar sinônimos, usar conectores do tipo OR, AND e outros. Nosso objetivo demanda uma buscas pilotos para refinar a proposta e achar estudos primários já conhecidos. Para obter esses primeiros trabalhos, a busca abarca só literatura em inglês.

Como resultado da estratégia supracitada foi definida a seguinte String de busca:

(Transparency AND Software) OR (Transparency AND Organizational) OR (Transparency AND Process) OR (Transparency AND Open Data) OR (Transparency AND Access)

A estratégia adotada para realizar um SMS para transparência segue as propostas do Zhang et al. (2011) e Wohlin (2014), a proposta é de realizar um processo iterativo descrito a partir da Figura 2, o QGS (Quasi Gold Standard) e o backward and forward 
snowballing, porém o String acima descrito deve ser refinado conforme estas duas estratégias e as iterações necessárias até achar os artigos que efetivamente respondam as perguntas de pesquisa inicialmente propostas, ou seja, até achar o Quasi Gold Standard.

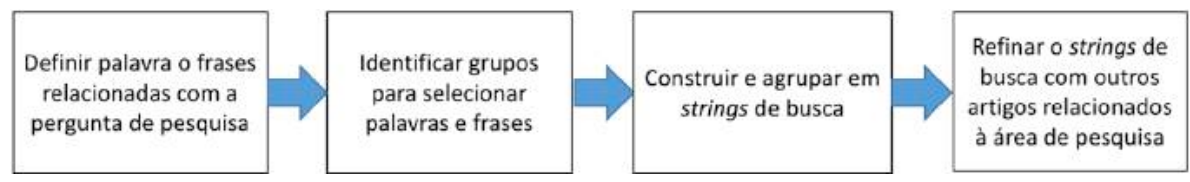

Figura 4. Processo de Seleção do String de Busca

\section{Resultados já Alcançados}

Na Tabela 3 são mostrados os resultados preliminares produzidos pela Biblioteca EAFIT com o String de busca acima descrito. Foram achados 36 artigos, dos quais foi aplicado o critério de exclusão por titulo e são apresentados na Tabela 3, 29 artigos.

Desse primeiro piloto foi identificado que não existe estudos secundários nem do tipo RSL ou EMS no contexto da Transparência ou mais especificamente Transparência de Software. Porem é necessário para a construção do conhecimento usar este tipo de estudos baseado em evidencias, mapas e trabalhos que permitam identificar de forma agrupada o que foi feito ou quais são os desertos ou oportunidades de pesquisa para direcionar trabalhos presentes e futuros na área.

Acreditamos que os artigos achados servem como ponto de partida para realizar a busca manual sugerida por Zhang et al. (2011), o piloto também segue as sugestões propostas por Wohlin (2014), que propõe o uso do Google Scholar, por exemplo, para identificar artigos base. Isso evita distorcer a favor de qualquer autor. Wohlin também recomenda: usar artigos relevantes de diferentes comunidades; o número de artigos no início deve ser pequeno, aumentar e depois ir para o um foco mais especifico. Além disso, sugere que desses artigos inicialmente identificados pode-se obter os termos de pesquisa para refinar a própria, e assim evitar se guiar unicamente o uso da própria terminologia e abranger mais trabalhos, bases de dados, autores, anos, entre outros. Em uma palavra obter mais diversidade.

\subsection{Discussão sobre os artigos achados:}

Por questões de espaço e do alcance desse trabalho não serão analisadas uma a uma as perguntas de pesquisa propostas para o EMS e que fazem parte do trabalho futuro descrito a seguir.

Essa primeira aproximação nos leva a utilização da definição de transparência fornecida pela Infopedia:" "diz-se de um termo, de um contexto ou de um discurso que não é opaco, que se percebe facilmente; evidente, claro e que revela claramente a sua natureza; inequívoco". Inspirados nessa definição os autores concordam em dizer que a transparência está ligada a algo suficientemente aberto para permitir que coisas sejam observadas em profundeza, através dela e por diferentes óticas. Quando é detalhado esse elemento a ser observado geralmente faz referencia á informação, aquela que deve informar sobre se mesma, como ela trabalha e seu rationale. Em outras palavras a

\footnotetext{
${ }^{1}$ https://www.infopedia.pt/dicionarios/lingua-portuguesa/Transparente
} 
definição de transparência é apresentada e proposta para levar em conta a dinâmica da produção de informação e as diferenças entre dados e informações A transparência visa reduzir ou eliminar os obstáculos relacionados com a falta de visibilidade ou opacidade, acessibilidade e entendimento da informação. Porem, quando a informação é transparente ela deve ter significado, ser verídica, e compreensível.

Então, como é produzida, elaborada e interpretada e para quem é certa informação? A resposta a essas questões envolve o processo, o software e o cidadão. $\mathrm{O}$ primeiro, relacionado a como a informação está sendo produzida, o software como o artefato ubíquo, que deve tirar o véu, que automatiza o processo e disponibiliza a informação para o cidadão, esse ultimo usará o software para se informar, avaliar e demandar seus direitos/ tomar decisões face à informação revelada.

Tabela 3. Motivação perguntas de pesquisa

\begin{tabular}{|c|c|c|}
\hline Num & Autores e ano & Artigo \\
\hline 1 & Bentol, J. C., Jaeger, P. T, \& Grimes, J. M. (2010) & Using ICTs to create a culture of transparency: E-govermment and social media as openness and anti-corruption tools for societies. \\
\hline 2 & Cappelli, C. (1), \& Leite, J. C. S. D. P. (2010) & Software transparency \\
\hline 3 & Cyras, V. (1), \& Lachmayer, F., (2), (2014) & Compliance and software transparency for the design of legal machines 275 \\
\hline 4 & Dabbish, L., Stuart, C., Tsay, J., \& Herbsleb, J. (2013) & Leveraging Transparency \\
\hline 5 & 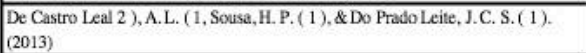 & Monitoring challenges of non-functional requirements: Evaluation in software transparency \\
\hline 6 & \begin{tabular}{|l} 
do Prado Leite, J. C. S., \& Cappelli, C. (2008) \\
\end{tabular} & Exploring i* Characteristics that Support Software Transparency \\
\hline 7 & Goctta, S., \& Davies, T. (2016) & The Daily Shaping of State Transparency: Standards, Machine-Readability and the Configuration of Open Government Data Policies \\
\hline 8 & Hase, K.-R. (2011) & $\begin{array}{l}\text { "Open Proof" for railway safety software - A potential way-out of vendor lock-in advancing to standardization, transparency, and } \\
\text { software security. }\end{array}$ \\
\hline 9 & Hocpman, J.H., \& Jacobs, B. (2007) & Icreased security Through Open Source \\
\hline 10 & Hosseini, M., Shahri, A, Phalp, K., \& Ali, R. (2016) & Foundations for transparency requirements engineering. \\
\hline 11 & Jansen, S. (1), Brinkkemper, S. (1), \& Finkelstein, A. (2), (2007) & Providing transparency in the business of software: A modeling technique for software supply networks. \\
\hline 12 & $\begin{array}{l}\text { Javadi,M.H.M. (1 ),Metrabi, J. ( 2), Beedel, M. ( } 3 \text { ).\& Tanhaei, M. H. ( } 4 \text { ). } \\
\text { (2012) }\end{array}$ & Effect of information systems and accounting softwares on transparency of financial statement information (FSI \\
\hline 13 & $\begin{array}{l}\text { José Albuquerque, D., Tavares Nunes, V., Ghedini Ralha, C., \& Cappelli, C. } \\
\text { (2017) }\end{array}$ & E-gov Transparency Implementation Using Multi-agent System; a Brazilian Study-Case in Lawsuit Distribution Process. \\
\hline 14 & Machill, M., Neuberger, C., \& Schindler, F. (2003) & Transparency on the Net: Functions and Deficiencies of Internet Search Engines. \\
\hline 15 & Martinez-Moyano, I. J. (2012) & Documentation for model transparency. \\
\hline 16 & Melo, C. de O., Ferraz, R., \& Parsons, R. J. (2016) & Brazil and the Emerging Future of Software Engineering. \\
\hline 17 & Meunier, P. (2008) & Software Transparency and Purity \\
\hline 18 & Michener, G., \& Bersch, K. (2011) & Conceptualizing the quality of transparency \\
\hline 19 & $\begin{array}{l}\text { Miller MN,D. (Minneapolis, Magnuson AZ), R. R. (Scottsdale, Barton NM), B. } \\
\text { J. (Albuquerque, \& Shao AZ), Q. G. (Oro V. (2011). }\end{array}$ & Two dimensional location transparency of software services. \\
\hline 20 & Pfleeger, C. P. (2016). & Looking into Software Transparency \\
\hline 21 & Powell, A. (2012). & Democratizing production through open source knowledge: from open sottware to open hardware. \\
\hline 22 & Reinhardt, W., \& Rinne, S. (2010a). & An Architecture to Support Learning, Awareness, and Transparency in Social Software Engineering. \\
\hline 23 & Rhod, E. L., Listoxa, C. A. L., Carro, L., Reorda, M. S., \& Violante, M. (n.d.). & Hardware and software transparency in the protection of programs against SEUs and SETs. \\
\hline 24 & Rojas, L. A. R., Lovelle, J. M. C., Bermúdez, G. M. T., \& Marín, C. E. M. (2013) & Open Data as a key factor for developing expert systems: a perspective from Spain. \\
\hline 25 & Serrano, M., \& do Prado Leite, J.C. S. (2011). & Capturing transparency-related requirements patterns through argumentation. \\
\hline 26 & Shull, F., Carleton, A., Carriere, J., Prikladnicki, R., \& Zhang, D. (2016) & The Future of Software Engineering. \\
\hline 27 & Tu, Y.-C., Tempero, E., \& Thomborson, C. (2016). & An experiment on the impact of transparency on the effectiveness of requirements documents \\
\hline 28 & Tu, Y.-C., Thomborson, C., \& Tempero, E. (2011). & Illusions and perceptions of transparency in software engineering \\
\hline 29 & Zinovatna, O.. \& Cysneiros. L. M. (2015). & Reusing knowledge on delivering privacy and transparcncy together \\
\hline
\end{tabular}

\subsection{Trabalho Futuro:}

Segundo o proposto de Kitchenham e Charters (2007) é necessário usar Strings de busca como ponto de partida. Porém buscas complementares são necessárias, por exemplo: listas de referência, literatura cinzenta, lugares de pesquisa específicos (revistas ou conferências) e pesquisadores da área. Os autores ressaltam que, infelizmente, a maioria de RLS ou EMS deixam de considerar buscas complementares.

É um desafio realizar a busca manual, dada a quantidade de trabalho que deve ser realizado. No entanto, acreditamos que é importante avaliar o uso e levantar as oportunidades de trabalho relacionadas a transparência.

Visando levantar essas oportunidades foram identificadas as seguintes tarefas: i) aumentar a robustez do trabalho realizando a busca manual; ii) realizar buscas das referencias bibliográficas declaradas nos estudos primários; iii) verificar pontualmente a 
falta de guias e estudos empíricos do tipo RLS e EMS no âmbito da transparência; iv) estender o protocolo a outros pesquisadores bem como a diferentes a novos engenhos de busca, na transparência; v) ampliar a busca manual a novas conferências e periódicos; vi) pesquisar a literatura em Espanhol e Português.

Apresentamos as primeiras evidencias do uso do protocolo guia para realizar um EMS apropriado para pesquisadores de engenharia de software, que tenham interesse no tópico da transparência. A motivação principal é que o EMS serve como meio de avaliar e interpretar as pesquisas disponíveis relevantes para uma pergunta de pesquisa. Um EMS para transparência nos levaria a sintetizar a evidencia através da construção do conhecimento dada pela combinação dos estudos primários achados. O protocolo usado visa apresentar uma avaliação usando um método já estabelecido e auditável, ajudando pesquisadores chegarem a uma compreensão conjunta do estado da transparência de software como área de pesquisa.

\section{Referências}

Basili, V. R., Caldiera, G., and Rombach, H. D. (2012). The goal question metric approach, 1994. Citado, 3,11 .

Basili, V. R., Selby, R. W., and Hutchens, D. H. (1986). "Experimentation in software engineering". IEEE Transactions on software engineering, (7), 733-743.

De Almeida Filho, A, T (2011). Um Mapeamento Sistemático de Mecanismos para Guiar Estudos Empíricos em Engenharia de Software. Dissertação de Mestrado. Universidade Federal de Pernambuco.

Descubridor Universidad EAFIT (2017). http://www.eafit.edu.co/biblioteca3/recursos-electronicos/basesdatos/Paginas/descubridor.aspx\#.WMG1OhhDmHo ultimo acceso 9 de março 2017.

Keele, S. (2007). Guidelines for performing systematic literature reviews in software engineering. In Technical report, Ver. 2.3 EBSE Technical Report. EBSE. sn.

Kitchenham B. and Charters S. (2007) "Guidelines for Performing Systematic Literature Reviews in Software Engineering", Tech. rep., Technical report, EBSE Technical Report EBSE-2007-01.

Kitchenham, B. A., Dyba, T., and Jorgensen, M. (2004). "Evidence-based software engineering". In Proceedings of the 26th international conference on software engineering (pp. 273-281). IEEE Computer Society.

Levy, Y., and Ellis, T. J. (2006). "A systems approach to conduct an effective literature review in support of information systems research". Informing Science: International Journal of an Emerging Transdiscipline, 9(1), 181-212.

Moura M (2008). Um benchmarking framework para avaliação da manutenibilidade de software orientado a aspectos. Master's thesis, University of Pernambuco, Recife, PE, Brasil. 1.1, 1.2.1, 2.2, 3.2 .

Petersen, K., Feldt, R., Mujtaba, S., and Mattsson, M. (2008). "Systematic Mapping Studies in Software Engineering". In EASE (Vol. 8, pp. 68-77).

Serviço de Buscas Universidade EAFIT http://www.eafit.edu.co/biblioteca/servicios/Paginas/busquedas-bibliograficas.aspx, último acesso 3 de maio de 17.

Wohlin, C. (2014). "Guidelines for snowballing in systematic literature studies and a replication in software engineering". In Proceedings of the 18th international conference on evaluation and assessment in software engineering (p. 38). ACM.

Yang, Z., Li, Z., Jin, Z., and Chen, Y. (2014). "A systematic literature review of requirements modeling and analysis for self-adaptive systems". In International Working Conference on Requirements Engineering: Foundation for Software Quality (pp. 55-71). Springer International Publishing.

Zhang, H., Babar, M. A., and Tell, P. (2011). "Identifying relevant studies in software engineering. Information and Software Technology", 53(6), 625-637. 